\title{
Studies suggest an association between maternal periodontal disease and pre-eclampsia
}

\author{
Is periodontal disease associated with pre-eclampsia?
}

\author{
Conde-Agudelo A, Villar J, Lindheimer $\mathbf{M}$. \\ Maternal infection and risk of pre-eclampsia: systematic review \\ and meta-analysis. Am J Obstet Gynecol 2008; 198:7-22.
}

In this systematic review, several types of infections are identified and investigated: urinary tract infection, periodontal disease, Chlamydia pneumoniae infection, HIV infection, malaria and other persistent bacterial and viral infections. Separate analyses were conducted for each of them. This summary review will only focus on the link between pre-eclampsia and periodontitis, which was just a part of the original systematic review.

Data Sources MEDLINE, EMBASE, POPLINE, CINAHL, LILACS (all from inception to June 30,2007), proceedings of international meetings on pre-eclampsia, bibliography of the retrieved articles, reviews, chapters in standard textbooks on hypertension in pregnancy, and contact with investigators involved in the field were used to identify relevant studies. No language restrictions were imposed.

Study selection Cohort, case-control or cross-sectional studies with original data that evaluated the association between maternal periodontal disease and pre-eclampsia were included. Cases were defined as women suffering from hypertension plus proteinuria, after 20 weeks' gestation.

Data extraction and synthesis Data were extracted from each study according to design, geographic location, sample size, gestational age when periodontal disease was diagnosed, definition and severity of pre-eclampsia, confounding factors controlled for, temporality of the association, and report of dose-response gradient. Studies included in the systematic review were also included in the meta-analysis if they reported Odds Ratio (OR) or Relative Risk (RR) estimates with their 95\% Confidence Intervals (Cls), or provided the information necessary to calculate them. Results from different reports were combined to produce a pooled OR according to the Mantel-Haenszel method, using both fixed-and random-effects models. Heterogeneity was quantified with $\mathrm{I}^{2}$ statistics. Studies were also quality assessed.

Results Seven case-control studies and 2 cohort studies evaluated the association between periodontal disease and pre-eclampsia. Six studies, representing a total of 3420 women (493 pre-eclamptic and 2927 nonpre-eclamptic control women) were pooled for meta-analysis. Women with evidence of periodontal disease during pregnancy had a 1.76 fold higher risk of pre-eclampsia compared with women without periodontal disease (OR, 1.76, 95\% Cl: 1.43-2.18).

Conclusions Periodontal disease during pregnancy is associated with an increased risk of pre-eclampsia. More studies are required to verify this as well as to explore whether or not such relationship is causal and, if so, the mechanisms involved.

Address for correspondence: Dr Conde-Agudelo, Perinatology Research Branch, Intramural Division, NICHD/NIH/DHHS, Baltimore, MD, USA

\section{Commentary}

This systematic review deals with the question of whether maternal infection - and periodontal disease in particular - is associated with pre-eclampsia, a multifactorial disorder that complicates about $3 \%$ of all pregnancies. Pre-eclampsia remains an important cause of maternal and perinatal morbidity and mortality in both developed and developing countries.

It is now well documented that periodontal diseases could have an impact on systemic illnesses, including atherosclerotic cardiovascular disease ${ }^{1}$. Given the link between cardiovascular disease and pre-eclampsia on the one hand ${ }^{2}$, and the association between periodontal disease and adverse pregnancy outcomes on the other ${ }^{3}$, it has recently been hypothesised that pre-eclampsia could be associated with periodontal disease.

Conde-Agudelo and colleagues have produced a good-quality systematic review. In spite of space limitations due to the investigation of multiple infections, results of the systematic review are highly reproducible, both in terms of search strategy and calculation of pooled OR. Results of the meta-analysis suggest that pre-eclampsia is associated with periodontal disease, with some evidence of a dose-response gradient. In addition, a recent Brazilian case-control study ${ }^{4}$, published after the present systematic review, provided quite a similar OR (OR=1.52; 95\% CI: 1.01 to 2.29$)$, adding strength to the hypothesis.

There are nevertheless some limitations to this systematic review that warrant consideration. As stated by the authors, even if adjustments for some confounding factors are made, residual confoundings remain a serious problem in observational studies. Second, the quality assessment method used in this systematic review seems not to be sufficiently discriminative, since the nine studies included are rated as "being of high-quality", which is obviously not the case. It would have been more appropriate to use the STROBE statement to assess the quality of reporting of observational studies ${ }^{5}$. Thus the result of the meta-analysis should be interpreted with caution, given that confounding factors and bias may account for a considerable proportion of the association.

Moreover, a recent randomised controlled trial found a non-significant increased risk of pre-eclampsia (OR=1.59, 95\% CI: 0.89 to 2.83 ) among pregnant women who received periodontal scaling and root planning before 21 weeks as compared to women who underwent scaling and root planning after delivery ${ }^{6}$. This could be explained by the fact that periodontal treatment has been shown to result in acute, short-term systemic inflammation and endothelial dysfunction ${ }^{7}$.

Finally, this systematic review provides another occasion to point out an important weakness in the current literature. Contrary to what Conde-Aguledo imply, using "the most stringent criteria for diagnosing periodontal disease (pocket depth, clinical attachment level, bleeding on probing and gingival inflammation)" is not, in itself, sufficient to guarantee that studies are comparing similar 
degrees of exposure to periodontal disease. As stated by D. Matthews in a recent commentary for $\mathrm{EBD}^{8}$, "there is a lack of a standardised protocol to determine periodontal diseases. The research community needs to address this issue and develop consensus".

\section{Practice point:}

To date, observational studies have suggested an association between maternal periodontal disease and pre-eclampsia. It has also been shown that treatment of periodontitis in pregnant women alleviates periodontal disease but, according to current evidence, it would be presumptuous to treat pregnant women in the aim of reducing the risk of pre-eclampsia.

\section{Jean-Noel Vergnes}

Toulouse Dental Hospital, Dental Department of Epidemiology, and Unit on Perinatal and Women's Health, Institut National de la Santé et de la Recherche Médicale (INSERM), U149, Paris, France
1 Bahekar AA, Singh S, Saha S, Molnar J, Arora R. The prevalence and incidence of coronary heart disease is significantly increased in periodontitis: a meta-analysis. Am Heart J 2007; 154:830-837.

2 Bellamy L, Casas JP, Hingorani AD, Williams DJ. Pre-eclampsia and risk of cardiovascular disease and cancer in later life: systematic review and meta-analysis. BMJ 2007; 335(7627):974.

3 Vergnes JN, Sixou M. Preterm low birth weight and maternal periodontal status: a meta-analysis. Am J Obstet Gynecol 2007;196:135.e1-7.

4 Siqueira FM, Cota LO, Costa JE, Haddad JP, Lana AM, Costa FO. Maternal periodontitis as a potential risk variable for pre-eclampsia: a case-control study. J Periodontol 2008; 79:207-215.

5 von Elm E, Altman DG, Egger M, Pocock SJ, Gøtzsche PC, Vandenbroucke JP; STROBE Initiative. The Strengthening the Reporting of Observational Studies in Epidemiology (STROBE) statement: guidelines for reporting observational studies. PLoS Med 2007; 4:e296.

6 Michalowicz BS, Hodges JS, DiAngelis AJ, Lupo VR, Novak MJ, Ferguson JE, Buchanan W, Bofill J, Papapanou PN, Mitchell DA, Matseoane S, Tschida PA; OPT Study. Treatment of periodontal disease and the risk of preterm birth. N Engl J Med 2006; 355: 1885-1894.

7 Tonetti MS, D'Aiuto F, Nibali L, Donald A, Storry C, Parkar M, Suvan J, Hingorani AD, Vallance $P$, Deanfield J. Treatment of periodontitis and endothelial function. N Engl J Med 2007; 356:911-920.

8 Matthews D. Possible link between periodontal disease and coronary heart disease. Evid Based Dent 2008; 9:8.

Evidence-Based Dentistry (2008) 9, 46-47. doi:10.1038/sj.ebd.6400580

\section{Key to evidence graphic used in the Evidence-based Dentistry Journal}

The graphic is based on the Centre for Evidence-based Medicine levels of Evidence tables www.cebm.net/levels_of_evidence.asp (see Evidence-based Dentistry 2003;4: p 17-18)

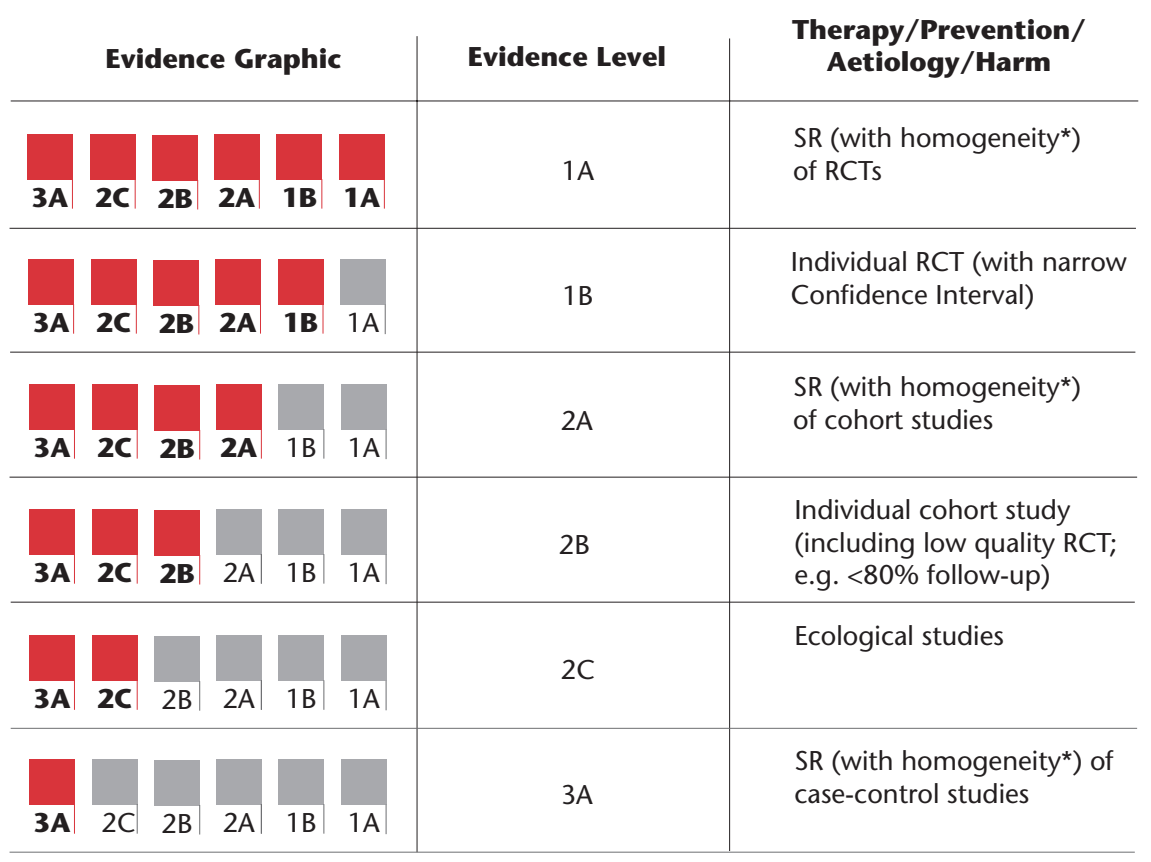

* By homogeneity we mean a systematic review that is free of worrisome variations (heterogeneity) in the directions and degrees of results between individual studies. Not all systematic reviews with statistically significant heterogeneity need be worrisome, and not all worrisome heterogeneity need be statistically significant. 